\title{
Comparison of TomoTherapy and RapidArc in Hippocampus Sparing Brain Radiotherapy in Pediatrics
}

\author{
Mohamed Nazmy ${ }^{1,2}$, Amr Mousa $^{2 *}$, GhadeerNazer ${ }^{3}$, Belal Moftah ${ }^{3}$ and Yasser Khafaga ${ }^{4}$ \\ ${ }^{1}$ Department of Radiation Oncology, Saint Luke's Cancer Center, Dublin, Ireland \\ ${ }^{2}$ Department of Radiation Oncology, National Cancer Institute, Cairo University, Cairo \\ ${ }^{3}$ Department of Biomedical Physics, Research Center, King Faisal Specialist Hospital and Research center Medical, Riyadh, Saudi Arabia \\ ${ }^{5}$ Radiation Oncology Section, Oncology Center, King Faisal Specialist Hospital and Research Center, Riyadh, Saudi Arabia
}

*Corresponding author: Amr Mousa, Department of Oncology, Oncology Center, Radiation Oncology section, King Faisal specialist Hospital and Research center, Riyadh, Saudi Arabia. Tel: 009966500645962; E-mail: amrgabermousa@yahoo.com

Received date: January 16, 2017; Accepted date: January 20, 2017; Published date: January 23, 2017

Copyright: (C) 2017 Nazmy M, et al. This is an open-access article distributed under the terms of the Creative Commons Attribution License, which permits unrestricted use, distribution, and reproduction in any medium, provided the original author and source are credited.

\begin{abstract}
Background and Objectives: Both TomoTherapy and RapidArc have been examined in hippocampus sparing whole brain irradiation in adults. We aim to compare both techniques in pediatrics with relatively smaller brain volume.

Patients and Methods: The hippocampus, the whole brain and the eyes were manually contoured in 3 pediatric patients. TomoTherapy plan was created aiming at adequate brain coverage and as low as possible hippocampus dose, RapidArc was challenged to achieve similar or better plan results. The prescription dose was 36 Gy in 20 treatment fractions to the whole brain.
\end{abstract}

Results: TomoTherapy was able to achieve a mean hippocampus dose of 13.6 Gy with brain homogeneity index [HI] of 0.14 Using RapidArc [single arc], the same hippocampus dose was not achievable without compromising the brain $\mathrm{HI}$ significantly. By using 2 arcs, similar results to the TomoTherapy plan were achieved. The treatment time for TomoTherapy was 3.5 minutes while it was 1.2 minutes for single arc and 2.4 minutes for two arcs.

Conclusions: TomoTherapy can achieve better target coverage with lower doses to the hippocampus compared to single arc RapidArc technique, while using 2 arcs RapidArc can achieve similar results with shorter treatment time.

Keywords: TomoTherapy; RapidArc; Hippocampus; Pediatrics

\section{Introduction}

The use of whole brain radiation [WBRT] with hippocampus sparing to preserve the neurocognitive functions is a novel concept that has been explored recently in patients with brain metastasis. This was based on data showing low incidence of limbic system involvement by brain metastasis [1]. It has been evaluated in adult patients using TomoTherapy [2] and RapidArc (RA) [3]. Central nervous system [CNS] tumors are the commonest solid pediatric malignancy with whole brain irradiation commonly given to pediatric patients either alone or as part of craniospinal irradiation [CSI]. The side effects of radiation on the neurocognitive functions are of significant concern in young patients [4,5]. In March 2009 both RapidArc and TomoTherapy facilities started to run clinically in our center, and the cumulative training and experience of the staff in both techniques are nearly the same.

Few data exist regarding direct comparison of TomoTherapy and RapidArc from centers with equal cumulative experience in both techniques. And data regarding hippocampus sparing is nearly absent in pediatric patients with relatively small brain volume. The aim of this study is to compare TomoTherapy and RapidArc in hippocampus sparing WBRT technique in pediatric patients regarding the mean hippocampus dose, target homogeneity index $[\mathrm{HI}]$ and treatment time.

\section{Patients and Methods}

After revision and approval of the study by the research committee, three pediatric patients with the diagnosis of cerebellar medulloblastoma were chosen. They were actually treated with 3-D conformal CSI technique. We investigated the feasibility of using TomoTherapy and RapidArc to deliver WBRT with hippocampus sparing on virtual plans. For the purpose of actual treatment planning, the patients were scanned by a wide bore system computed tomography $[\mathrm{CT}]$ using the axial scan mode. The CT scan implemented a $2 \mathrm{~mm}$ slice thickness over the entire head region. Each patient also underwent a T1- weighted, post-contrast axial magnetic resonance imaging [MRI] scan. The anatomic contours were delineated on the fused CT and magnetic resonance image sets in the treatment planning system [Eclipse].

The hippocampus, the whole brain and the eyes were manually contoured. A $5 \mathrm{~mm}$ volumetric margin expansion was applied to the hippocampus. The CTV for the brain included the whole brain tissues and meninges defined at the inner table of the skull after subtracting the hippocampus with the $5 \mathrm{~mm}$ expansion volume. Planning Target Volume [PTV] was defined as CTV plus $3 \mathrm{~mm}$ margin. All the three 
patients were re-planned twice, for the purpose of dosimetric comparison between RA and TomoTherapy. The first plan was done using TomoTherapy aiming at adequate brain coverage and as low as possible hippocampus dose. We hypothesized that RapidArc could achieve similar or better results. Both planning were done using the same normal structure and target contours as defined on CT-MRI fusion.

The TomoTherapy planning technique was done as prescribed by Gutierrez et al. [2]. The CT images and associated contours were transferred to the TomoTherapy Treatment Planning System [TPS] from the Eclipse TPS using the digital imaging and communications on medicine [DICOM]-RT protocol. For this study, plans were generated using a $2.5 \mathrm{~cm}$ FW to obtain the most conformal treatment plan deliverable in a reasonable period. For all plans, the modulation factor was set to $3.5 \mathrm{~cm}$.

The RapidArc planning technique was done as prescribed by Hsu et al. [3]. The CT planning images and associated contours were transferred to the RapidArc optimization planning system environment in the Eclipse treatment planning system using the DICOM format. Using the RapidArc optimization algorithm, RapidArc treatment plans were generated for WBRT with conformal avoidance of the hippocampi. The RapidArc plans consist of an arc, starting at a gantry angle of 179 degrees and rotating counterclockwise through 359 degrees to stop at a gantry angle of 181 degrees. During optimization, multileaf collimator [MLC]-shaped fields are progressively added throughout the arc. The gantry rotation speed and monitor units $[\mathrm{MU}]$ per gantry angle degree was optimized for a variable dose rate plan with a maximum dose rate of $600 \mathrm{MU} / \mathrm{min}$. in the first phase of the study planning was done using single arc, but after failure of achieving comparable results with TomoTherapy, 2 arcs have been used. The delivery time was calculated for each of the three plans [TomoTherapy, RapidArc single arc, and Rapid Arc 2 arcs].

\section{Dose prescription and dose constrains}

The main planning objective was to reduce the mean dose for the hippocampus as much as possible without compromising the coverage of the target volume. The dose of $36 \mathrm{~Gy}$ was prescribed to $95 \%$ of the volume of the whole brain [PTV], and delivered in 20 treatment fractions. The dose constraint applied to the eye was that no more than $50 \%$ of the eyes would receive $>5 \mathrm{~Gy}$. At the commencement of the trial a very hard dose constrains were applied aiming to achieve a comparable results with previous publications [3] namely, to reduce the mean dose for the hippocampi to $<6 \mathrm{~Gy}$ without compromising on coverage of the whole brain. As we progressed in the planning in both TomoTherapy and RapidArc, it was clear that these results cannot be achieved so the constrain was loosen during the optimization process based on the dosimetrist experience to achieve the best compromise between both.

\section{Plans comparison}

The treatment plan metrics were compared using the following parameters: HI, the mean and the maximum hippocampus dose. Dose homogeneity in the target volumes were quantified by the HI, as recommended by the International Commission on Radiation Units and Measurements [6]. The $\mathrm{HI}$ is defined as the greatest dose delivered to $2 \%$ of the target volume [D2\%] minus the dose delivered to $98 \%$ of the target volume [D98\%] divided by the median dose [Dmedian] of the target volume:

\section{$\mathrm{HI}=\mathrm{D} 2 \%-\mathrm{D} 98 \% /$ Dmedian}

Smaller values of HI correspond to more homogenous irradiation of the target volume. A value of 0 corresponds to absolute homogeneity of dose within the target. The statistical package for social sciences [SPSS] version 15 was used for data analysis.

\section{Ethical consideration}

This is a comparative dosimetric planning study. All planning were virtually done on CT images stored on our planning database. No comparison or analysis was done regarding any of the actual delivered treatment.

\section{Results}

The average age of the studied patients was 6.3 years. The mean volume of the brain PTV was 1377 cc while the mean hippocampus volume was $1.7 \mathrm{cc}$. After several trials with single arc RapidArc planning it was clear from the data of the three patients that we cannot achieve comparable results to TomoTherapy, so a third plan was added and compared using 2 Arcs. The goal for the eye was achieved for all patients in all the three plans with no more than $50 \%$ of the eyes receiving $>5$ Gy. Table 1 summarizes the patients' characteristics and the planning results for the three studied patients.

\begin{tabular}{|c|c|c|c|c|c|c|c|c|c|}
\hline Patient & Age & Hipp vol. (cc) & Brain vol. (cc) & Technique & $\begin{array}{l}\text { HippMean } \\
\text { (Gy) }\end{array}$ & $\begin{array}{l}\text { Hipp Max } \\
\text { (Gy) }\end{array}$ & $\begin{array}{l}\text { D2 } \\
\text { (Gy) }\end{array}$ & $\begin{array}{l}\text { D98 } \\
\text { (Gy) }\end{array}$ & HI \\
\hline \multirow{3}{*}{1} & \multirow{3}{*}{10} & \multirow{3}{*}{2} & \multirow{3}{*}{1290} & Tomotherapy & 13.6 & 25.8 & 39.1 & 34.8 & 0.11 \\
\hline & & & & Single arc & 14.9 & 22.9 & 39.8 & 33.3 & 0.17 \\
\hline & & & & Two arcs & 13.4 & 17.9 & 38.5 & 34.4 & 0.11 \\
\hline \multirow{3}{*}{2} & \multirow{3}{*}{5} & \multirow{3}{*}{2.2} & \multirow{3}{*}{1447} & Tomotherapy & 12.8 & 25.1 & 39.1 & 32.3 & 0.18 \\
\hline & & & & Single arc & 13.6 & 25.5 & 42 & 30.4 & 0.29 \\
\hline & & & & Two arcs & 12.8 & 19.5 & 39.6 & 31.3 & 0.2 \\
\hline 3 & 4 & 1 & 1394 & Tomotherapy & 14 & 29 & 39.5 & 34.4 & 0.13 \\
\hline
\end{tabular}


Citation: $\quad$ Nazmy M, Mousa A, Nazer G, Moftah B, Khafaga Y (2017) Comparison of TomoTherapy and RapidArc in Hippocampus Sparing Brain Radiotherapy in Pediatrics. J Nucl Med Radiat Ther 8: 326. doi:10.4172/2155-9619.1000326

Page 3 of 5

\begin{tabular}{|c|c|c|c|c|c|c|c|c|c|}
\hline & & & & Single arc & 14.9 & 23.6 & 40.2 & 34.6 & 0.15 \\
\hline & & & & Two arcs & 14.5 & 29.2 & 39.2 & 34.5 & 0.13 \\
\hline \multirow{3}{*}{ Average } & \multirow{3}{*}{6.3} & \multirow{3}{*}{1.7} & \multirow{3}{*}{1377} & Tomotherapy & 13.6 & 27.5 & 39.2 & 33.8 & 0.14 \\
\hline & & & & Single arc & 14.5 & 24 & 40.6 & 32.7 & 0.2 \\
\hline & & & & Two arcs & 13.6 & 22.2 & 39.1 & 33.4 & 0.14 \\
\hline
\end{tabular}

Table 1: Data of the patients comparing TomoTherapy with RapidArc "single arc and two arcs".

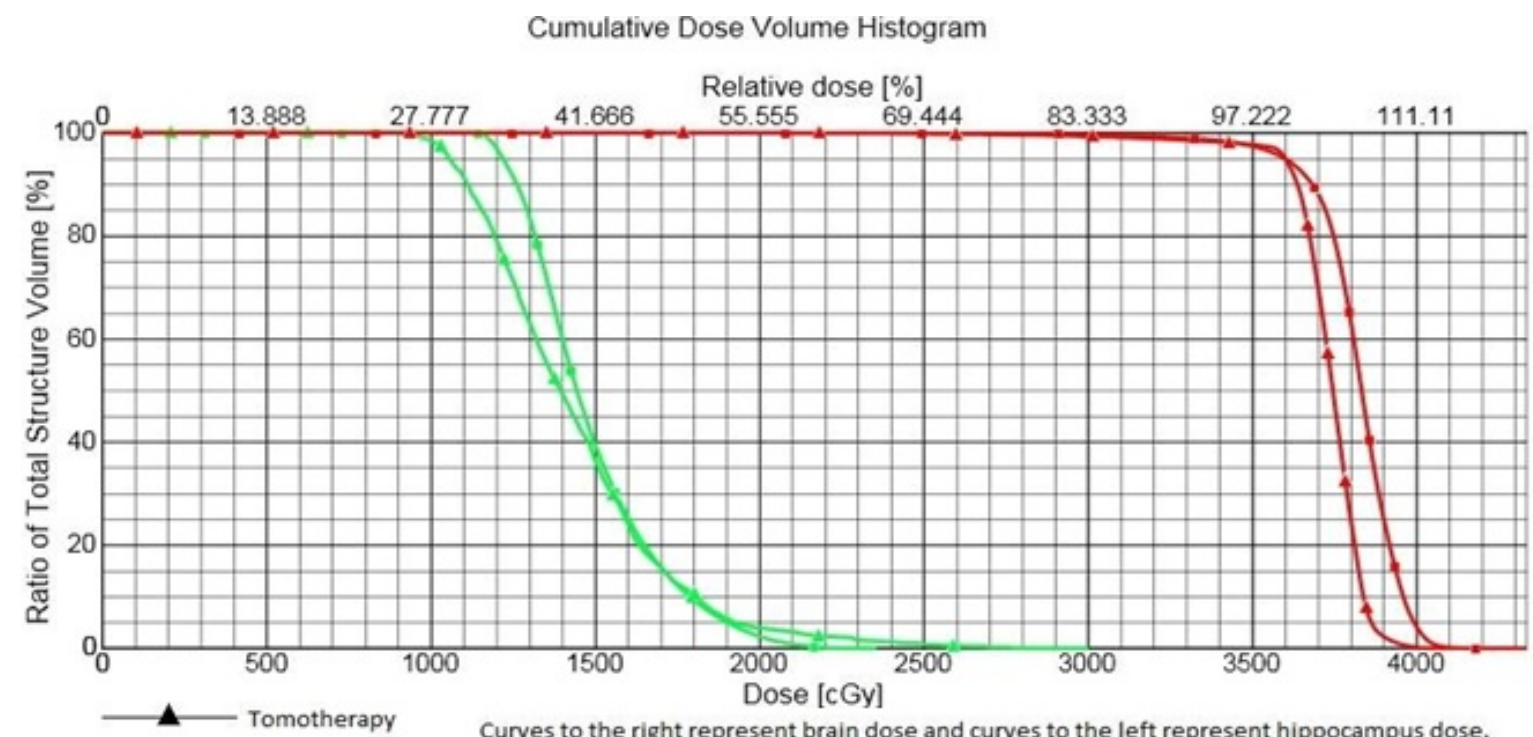

Figure 1: DVH comparing TomoTherapy to one arc (Cumulative Dose Volume Histagram).

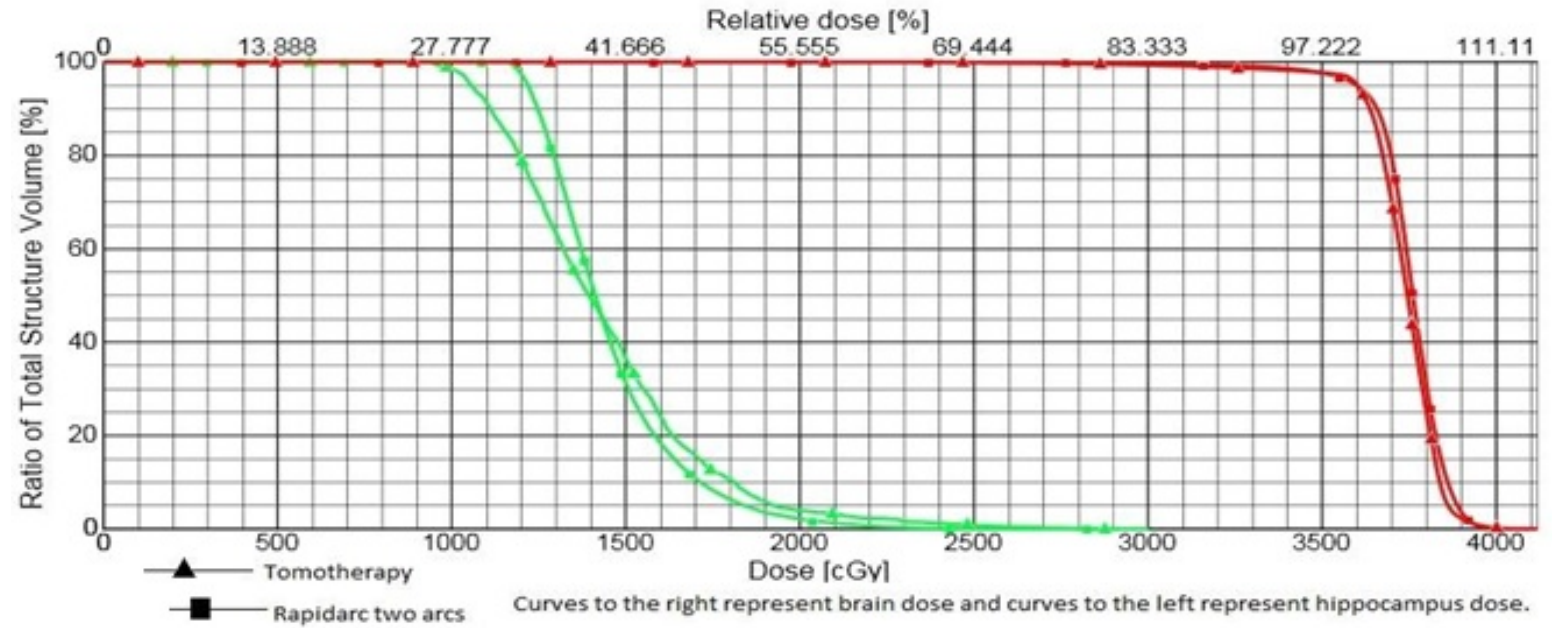

Figure 2: DVH comparing TomoTherapy to two arcs (Cumulative Dose Volume Histagram).

TomoTherapy was able to achieve a mean hippocampus dose of 13.6 Gy with HI of 0.14. With RapidArc [singlearc] the same hippocampus dose was not achievable without compromising the HI significantly, with mean hippocampus dose of $14.5 \mathrm{~Gy}$, the HI was 0.20 By using 2 
Page 4 of 5

arcs, similar doses to the TomoTherapy plan were archived, namely, a mean hippocampus dose of 13.6 Gy with $\mathrm{HI}$ of 0.14 . Figure 1 shows the DVH of one patient comparing TomoTherapy to one arc and Figure 2 shows the DVH of one patient comparing TomoTherapy to two arcs. The treatment time for TomoTherapy was 3.5 minutes while it was 1.2 minutes for single arc and 2.4 minutes for two arcs.

\section{Discussion}

TomoTherapy and RapidArc are relatively new volumetric modulated arc therapy that delivers radiation through arc technique. The RapidArc technique has shown superiority [better conformity, more favorable dose distributions, shorter treatment times and better normal tissue sparing] compared to conventional IMRT in different treatment sites like Head \& Neck [7] Brain [8] Pelvis [9] and breast tumors [10]. Also TomoTherapy showed a competitive superiority results when compared with IMRT in several sites. [11-15]. Rong et al [16] conducted a joint trial between 2 centers, University of Maryland and University of Wisconsin Cancer Center in order to compare both techniques. It showed that in different treatment sites both the treatment delivery time and the total MUs are less with RapidArc than with TomoTherapy, while the target dose uniformity is slightly better with TomoTherapy.

Whole brain RT with hippocampus sparing and simultaneous integrated boost [SIB] using either RapidArc or TomoTherapy has been explored in adult cancer patients with brain metastases. This technique was described to achieve homogeneous WBRT, with a high conformality to the metastatic lesions, while limiting the hippocampus dose to $<6 \mathrm{~Gy}$.

In the current study we explored the use of WBRT hippocampus sparing techniques in pediatric patients with medulloblastoma. Patterns of failure of medulloblastoma suggest that supratentorial metastases/relapses involve the meninges, sub-temporal, sub-frontal, the ventricles or the hypothalamic areas, while relapses in the hippocampus area are nearly nonexistent [17].

The number of the studied patients was limited to 3 as the results achieved with TomoTherapy compared to RapidArc [single or two arcs] were consistent among the three studied patients. We may come up with a follow up article in the future with more number and robust results.

In the current study, neither TomoTherapy nor RapidArc was able to achieve a low dose to the hippocampus with good target coverage of the brain in pediatrics as what has been achieved in previous publications in adults [3]. One reason for this could be the relative small size of the brain in pediatrics.

Whole brain irradiation is part of many pediatric brain tumor protocols. Most of the trials are exploring the reduction of the total dose or the field size as the feasibility of reducing the dose to a specific area during brain irradiation is a novel technology achievement [18]. A recently published trial compared different techniques like intensitymodulated radiotherapy (IMRT), intensity-modulated arc therapy (IMAT), and intensity-modulated proton therapy (IMPT) in reducing hippocampus dose in pediatrics with more strict criteria for brain coverage, estimated risks for developing memory impairment to be $33 \%$ with limiting the hippocampus dose to $10 \mathrm{~Gy}$ and $41 \%$ with $18 \mathrm{~Gy}$ and advised testing pediatric hippocampus sparing in a prospective clinical trial [19].

\section{Conclusions}

For whole brain radiation, TomoTherapy can achieve better target coverage with lower dose to the hippocampus as compared to single arc RapidArc technique, while using 2 arcs can achieve similar results with shorter overall treatment time. Centers having both facilities would better use RapidArc ( 2 arcs) for hippocampus sparing brain irradiation in pediatrics to decrease the treatment duration.

\section{References}

1. Ghia A, Tomé WA, Thomas S, Cannon G, Khuntia D, et al. (2007) Distribution of brain metastases in relation to the hippocampus: implications for neurocognitive functional preservation. Int $\mathrm{J}$ Radiat Oncol Biol Phys 68: 971-977.

2. Gutiérrez AN, Westerly DC, Tomé WA, Jaradat HA, Mackie TR, et al. (2007) Whole brain radiotherapy with hippocampus avoidance and simultaneously integrated brain metastases boost: a planning study. Int J Radiat Oncol Biol Phys 69: 589-597.

3. Hsu F, Carolan H, Nichol A, Cao F, Nuraney N, et al. (2010) Whole brain radiotherapy with hippocampus avoidance and simultaneous integrated boost for 1-3 brain metastases: a feasibility study using volumetric modulated arc therapy. Int J Radiat Oncol Biol Phys. 76: 1480-1485.

4. Grill J, Renaux VK, Bulteau C, Viguier D, Piebois LC, et al. (1999) Longterm intellectual outcome in children with posterior fossa tumors according to radiation doses and volumes. Int J Radiat Oncol Biol Phys. 45: 137-145.

5. Watanabe S, Azami Y, Ozawa M, Kamiya T, Hasegawa D, et al. (2011) Intellectual development after treatment in children with acute leukemia and brain tumor. Pediatr Int 53: 694-700.

6. http://www.icru.org/home/reports/prescribing-recording-and-reportingphoton-beam-therapy-report-62

7. Vanetti E, Clivio A, Nicolini G, Fogliata A, Laskar GS, et al. (2009) Volumetric modulated arc radiotherapy for carcinomas of the oropharynx, hypo-pharynx and larynx: a treatment planning comparison with fixed field IMRT. Radiother Oncol 92: 111-117.

8. Wagner D, Christiansen H, Wolff H, Vorwerk H (2009) Radiotherapy of malignant gliomas: comparison of volumetric single arc technique (RapidArc), dynamic intensity-modulated technique and 3D conformal technique. Radiother Oncol 93: 593-596.

9. Cozzi L, Dinshaw KA, Shrivastava SK, Mahantshetty U, Engineer R, et al (2008) A treatment planning study comparing volumetric arc modulation with RapidArc and fixed field IMRT for cervix uteri radiotherapy. Radiother Oncol 89: 180-191.

10. Qiu JJ, Chang Z, Wu QJ, Yoo S, Horton J, et al. (2010) Impact of volumetric modulated arc therapy technique on treatment with partial breast irradiation. Int J Radiat Oncol Biol Phys 78: 288-296.

11. Lee TF, Fang FM, Chao PJ, Su TJ, Wang LK, et al. (2008) Dosimetric comparisons of helical TomoTherapy and step-and-shoot intensitymodulated radiotherapy in nasopharyngeal carcinoma. Radiother Oncol 89: 89-96.

12. Yartsev S, Kron T, Cozzi L, Fogliata A, Bauman G (2005) TomoTherapy planning of small brain tumours. Radiother Oncol.74: 49-52.

13. Rodrigues G, Yartsev S, Chen J, Wong E, D'Souza D, et al (2006) A comparison of prostate IMRT and helical TomoTherapy class solutions. Radiother Oncol 80: 374-377.

14. Cattaneo GM, Delloca I, Broggi S, Fiorino C, Perna L, et al. (2008) Treatment planning comparison between conformal radiotherapy and helical TomoTherapy in the case of locally advanced-stage NSCLC Radiother Oncol 88: 310-318.

15. Caudrelier JM, Morgan SC, Montgomery L, Lacelle M, Nyiri B, et al. (2009) Helical TomoTherapy for locoregional irradiation including the internal mammary chain in left-sided breast cancer: dosimetric evaluation. Radiother Oncol 90: 99-105. 
Citation: Nazmy M, Mousa A, Nazer G, Moftah B, Khafaga Y (2017) Comparison of TomoTherapy and RapidArc in Hippocampus Sparing Brain Radiotherapy in Pediatrics. J Nucl Med Radiat Ther 8: 326. doi:10.4172/2155-9619.1000326

Page 5 of 5

16. Rong Y, Tang G, Welsh JS, Mohiuddin MM, Paliwal B, et al. (2011) Helical TomoTherapy versus single-arc intensity-modulated arc therapy: a collaborative dosimetric comparison between two institutions. Int J Radiat Oncol Biol Phys. 81: 284-296.

17. Miralbell R, Bleher A, Huguenin P, Ries G, Kann R, et al (1997) Pediatric medulloblastoma: radiation treatment technique and patterns of failure. Int J Radiat Oncol Biol Phys 37: 523-529.
18. Gardas SA (2009) A literature review of the recent radiotherapy clinical trials in pediatric brain tumors. Rev Recent Clin Trials 4: 42-55.

19. Blomstrand M, Brodin NP, MunckAfRosenschöld P, Vogelius IR, Merino SG, et al. (2012) Estimated clinical benefit of protecting neurogenesis in the developing brain during radiation therapy for pediatric medulloblastoma. Neuro Oncol 14: 882-889. 